\title{
Short-Range Interaction Impact on Two-Dimensional Dipolar Scattering
}

\author{
Eugene A. Koval ${ }^{1,2, \star}$ and Oksana A. Koval ${ }^{3, \star \star}$ \\ ${ }^{1}$ Bogoliubov Laboratory of Theoretical Physics, Joint Institute for Nuclear Research, Dubna, Moscow Region \\ 141980, Russia \\ ${ }^{2}$ Department of fundamental problems of micro-world physics, Dubna University, Dubna, Moscow Region, \\ Russia \\ ${ }^{3}$ Obukhov Institute for Atmospheric Physics, Moscow, Russia
}

\begin{abstract}
We report numerical investigation of the short range interaction influence on the two-dimensional quantum scattering of two dipoles. The model simulates two ultracold polar molecules collisions in two spatial dimensions. The used algorithm allows us to quantitatively analyse the scattering of two polarized dipoles with account for strongly anisotropic nature of dipolar interaction. The strong dependence of the scattering total cross section on the short range interaction radius was discovered for threshold collision energies. We also discuss differences of calculated scattering cross section dependencies for different polarisation axis tilt angles.
\end{abstract}

\section{Introduction}

In recent years, two-dimensional (2D) systems with anisotropic interactions have been of increasing interest. One can mention a gas of dipolar atoms or ultracold polar molecules in quasi-2D geometry of magneto-optical traps [1] and dipolar Rydberg atoms [2]. Beside the gas of polar molecules long range dipolar interaction also arises in a gas of 2D dipolar excitons in 2D quantum well of semiconductor heterostructures. Dipolar exciton gas quantum dynamics [3-5] and Bose-Enstein Condensate [6] have been also intensively investigated in recent years. Investigation of the dipolar diatomic molecules are also actual due to the prospects of their possible applications as cubits for the quantum computing [7].

Dipole-dipole interactions are long-range and strongly anisotropic, that leads to the possibility of the creation of exotic long-range quantum systems with dipolar gases [8, 9]. Particularly, anisotropic superfluidity [10], 2D dipolar fermions [11] and dipolar few-body complexes [12] have been investigated. For a recent progress in the physics of few-body systems with long-range interactions we refer the reader to the comprehensive review [13].

\section{Two-dimensional dipole-dipole scattering}

We model the polar molecules collisions in a layer of a pancake-shaped optical trap with dipoledipole scattering in two spatial dimensions. The quantum scattering in the plane is described by the

\footnotetext{
^e-mail: e-cov@yandex.ru

$\star \star$ e-mail: kov.oksana20@gmail.com
} 
2D Schrödinger equation in polar coordinates $(\rho, \phi)$

$$
\left[-\frac{\hbar^{2}}{2 \mu}\left(\frac{1}{\rho} \frac{\partial}{\partial \rho}\left(\rho \frac{\partial}{\partial \rho}\right)+\frac{1}{\rho^{2}} \frac{\partial^{2}}{\partial \phi^{2}}\right)+U(\rho, \phi)\right] \Psi(\rho, \phi)=E \Psi(\rho, \phi)
$$

with the scattering boundary conditions in the asymptotic region $\rho \rightarrow \infty$ :

$$
\Psi(\rho, \phi) \rightarrow e^{i q \rho}+f\left(q, \phi, \phi_{q}\right) \frac{e^{i q \rho}}{\sqrt{-i \rho}} .
$$

The interaction potential $U(\rho, \phi)$ reads

$$
\begin{gathered}
U(\rho, \phi)=V_{S R}(\rho)+V_{d d}(\rho, \phi), \\
V_{S R}(\rho)=\left\{\begin{array}{l}
\infty, \rho \leqslant \rho_{S R}, \\
0, \rho>\rho_{S R},
\end{array}\right.
\end{gathered}
$$

where the isotropic short-range potential $V_{S R}(\rho)$ is modeled by an infinitely high potential barrier at the origin, that was used in a number of papers of other the authors (see [14]). $V_{d d}(\rho, \phi)$ denotes the long-range and strongly anisotropic interaction potential of two arbitrarily oriented dipoles:

$$
V_{d d}(\rho, \phi ; \alpha, \beta, \gamma)=\frac{d_{1} d_{2}}{\rho^{3}}[\sin (\alpha) \sin (\gamma) \cos (\beta)+\cos (\alpha) \cos (\gamma)-3 \sin (\alpha) \sin (\gamma) \cos (\phi) \cos (\phi-\beta)],
$$

where $\boldsymbol{d}_{i},(i=1,2)$ are dipole moments. The angles $\alpha, \gamma$ denote to the tilt of each dipole $\boldsymbol{d}_{1}, \boldsymbol{d}_{2}$ with respect to the $Z$ axis (that is perpendicular to the plane of the motion) and $\beta$ is the angle between the dipoles polarisation planes $Z \boldsymbol{Z} \boldsymbol{d}_{1}, Z \boldsymbol{d}_{2}$ (see Ref. [15]).

If scattering amplitude is calculated the differential scattering cross section and the total cross section are defined as

$$
\sigma(q)=\frac{1}{2 \pi} \int_{0}^{2 \pi} \int_{0}^{2 \pi} \frac{d \sigma}{d \Omega} d \phi_{q} d \phi \equiv \frac{1}{2 \pi} \int_{0}^{2 \pi} \int_{0}^{2 \pi}\left|f\left(q, \phi, \phi_{q}\right)\right|^{2} d \phi_{q} d \phi,
$$

where $d \Omega=d \phi d \phi_{q}$ and the angle $\phi_{q}$ defines the direction $\boldsymbol{q} / q$ of the incident wave.

To integrate the problem (1)-(2) we use the algorithm applied in Refs. [15, 16] for the anisotropic quantum scattering in two dimensions and in Refs. $[17,18]$ for the investigation of bound state properties of 2D Hydrogen atom and 2D exciton in a tilted magnetic field. Following the ideas of the method suggested in Ref. [19] we choose the eigenfunctions $\xi_{m}(\phi)=e^{i m(\phi-\pi)} / \sqrt{2 \pi}=(-1)^{m} e^{i m \phi} / \sqrt{2 \pi}$ of the operator $h^{(0)}=\partial^{2} / \partial \phi^{2}$ as a Fourier basis for the angular-grid representation of the searched wave-function $\Psi(\rho, \phi)$. We introduce the uniform grid $\phi_{j}=2 \pi j /(2 M+1)($ where $j=0,1, \ldots, 2 M)$ over the $\phi$ - and $\phi_{q}$-variables and search the wave function as the expansion

$$
\Psi(\rho, \phi)=\frac{1}{\sqrt{\rho}} \sum_{j=0}^{2 M} \sum_{m=-M}^{M} \xi_{m}(\phi) \xi_{m j}^{-1} \psi_{j}(\rho)=\frac{1}{(2 M+1) \sqrt{\rho}} \sum_{j=0}^{2 M} \sum_{m=-M}^{M} e^{i m\left(\phi-\phi_{j}\right)} \psi_{j}(\rho),
$$

where $\xi_{m j}^{-1}=2 \pi \xi_{j m}^{*} /(2 M+1)=\sqrt{2 \pi} e^{-i m\left(\phi_{j}-\pi\right)} /(2 M+1)$ is the inverse matrix to the $(2 M+1) \times(2 M+1)$ square matrix $\xi_{j m}=\xi_{m}\left(\phi_{j}\right)$ defined on the angular grid.

This expansion reduces the 2D Schrödinger equation (1) to a system of $2 M+1$ coupled ordinary differential equations of the second order: 


$$
\frac{d^{2} \psi_{j}(\rho)}{d \rho^{2}}+\frac{2 \mu}{\hbar^{2}}\left(E-U\left(\rho, \phi_{j}\right)+\frac{\hbar^{2}}{8 \mu \rho^{2}}\right) \psi_{j}(\rho)+\frac{1}{\rho^{2}} \sum_{j^{\prime}} h_{j j^{\prime}}^{(0)} \psi_{j^{\prime}}(\rho)=0
$$

with the "left-side" boundary condition $\psi_{j}(\rho \rightarrow 0) \rightarrow$ const $\times \sqrt{\rho} \quad(j=0,1, \ldots, 2 M)$ and with the scattering boundary condition in the asymptotic region $\rho \rightarrow \infty$ of the form

$$
\frac{1}{(2 M+1) \sqrt{\rho}} \sum_{j=0}^{2 M} \sum_{m=-M}^{M} e^{i m\left(\phi-\phi_{j}\right)} \psi_{j}(\rho)=\exp \{i q \rho \cos (\phi)\}+f\left(q, \phi, \phi_{q}\right) \frac{e^{i q \rho}}{\sqrt{-i \rho}} .
$$

The key advantages of the algorithm are summarized below. Any local interaction in this representation is diagonal. In Ref. [15] much faster convergence over the angular grid nodes number than partial wave representation was proved. After the finite-difference approximation of the boundaryvalue problem (8-9) the obtained block matrix can be stored in a compressed form, which allows optimal resource using. The resulting algebraic system is efficiently solved by a fast implicit matrix algorithm based on the idea of the block sweep algorithm.

\section{Short-range interaction influence on 2D dipolar scattering}

The present study evidenced the strong dependence of the total cross section (6) on the short range cut-off radius $\rho_{S R}$ for threshold energies of dipole-dipole collisions. When the polarization axis is aligned along the $z$-axis the dipole-dipole interaction is isotropic and repulsive, no resonances occurs, because repulsive dipolar term prevents particles to getting too close to each other.

For a tilted $(\alpha=\gamma=0.25 \pi)$ polarization axis as well as for extremal case $(\alpha=\gamma=0.5 \pi)$ of polarization axis lying in the plane of particles motion, we discovered strong dependence of the total cross section on the cut-off radius $\rho_{S R}(4)$, which is illustrated in Fig. 1. The calculations were carried out at the energy $E=10^{-5}$ a.u. and a dipolar length $D=\mu d_{1} d_{2} / \hbar^{2}=1$. Note, that we study the case of distinguishable particles collisions.

The sharp increases in total cross section appear due to the virtual bound states arising as the interplay of the short-range (4) and long-range (5) potentials. It should be noted the existence of small cusps for the following values of $\rho_{S R}=0.035$ a.u., 0.0895 a.u., 0.42 a.u. For small values of $\rho_{S R}$ a resonance width decrease with simultaneous increase of their frequency are observed.

\section{Acknowledgements}

This work was supported by the Russian Foundation for Basic Research, Grant No. 16-32-00865.

\section{References}

[1] S. Ospelkaus, K.K. Ni, D. Wang, M. De Miranda, B. Neyenhuis, G. Quéméner, P. Julienne, J. Bohn, D. Jin, J. Ye, Science 327, 853 (2010)

[2] K. Afrousheh, P. Bohlouli-Zanjani, D. Vagale, A. Mugford, M. Fedorov, J.D.D. Martin, Phys. Rev. Lett. 93, 233001 (2004)

[3] R. Rapaport, G. Chen, S.H. Simon, Physical Review B 73, 033319 (2006)

[4] V.B. Timofeev, A.V. Gorbunov, A.V. Larionov, Journal of Physics: Condensed Matter 19, 295209 (2007)

[5] V. Timofeev, A. Gorbunov, Journal of applied physics 101, 081708 (2007) 

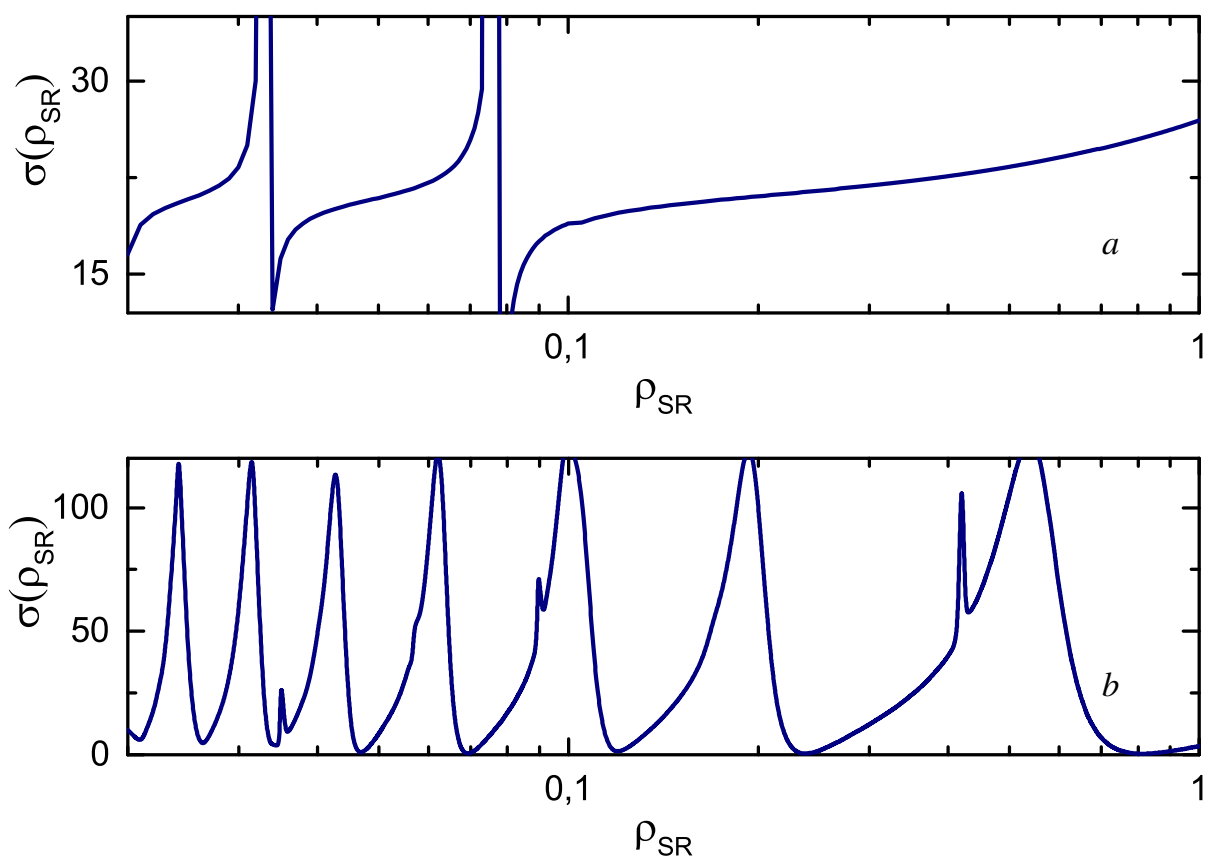

Figure 1. The full cross section $\sigma\left(\rho_{S R}\right)$ (6) dependence on the short range interaction radius $\rho_{S R}$ (4) for the case of polarized dipoles $\beta=0$ for $\alpha=\gamma=0.25 \pi$ (a) and $\alpha=\gamma=0.5 \pi$ (b) at the energy $E=10^{-5}$ a.u. and a dipolar length $D=\mu d_{1} d_{2} / \hbar^{2}=1$. The quantities are given in units $\hbar=\mu=1$.

[6] V. Timofeev, A. Gorbunov, Physica status solidi (c) 5, 2379 (2008)

[7] D. DeMille, Physical Review Letters 88, 067901 (2002)

[8] M.A. Baranov, Phys. Rep. 464, 71 (2008)

[9] I. Bloch, J. Dalibard, W. Zwerger, Reviews of modern physics 80, 885 (2008)

[10] C. Ticknor, Physical Review A 84, 032702 (2011)

[11] G.M. Bruun, E. Taylor, Physical review letters 101, 245301 (2008)

[12] J.C. Cremon, G. Bruun, S. Reimann, Physical review letters 105, 255301 (2010)

[13] Y. Wang, P. Julienne, C.H. Greene, Few-body physics of ultracold atoms and molecules with long-range interactions (World Scientific, 2015), chap. Chapter 2, pp. 77-134

[14] K. Kanjilal, D. Blume, Physical Review A 73, 060701 (2006)

[15] E.A. Koval, O.A. Koval, V.S. Melezhik, Physical Review A 89, 052710 (2014)

[16] E. Koval, O. Koval, V. Melezhik, Physics of Particles and Nuclei Letters 12, 448 (2015), [Pis'ma v Zh. Fiz. Elem. Chastits At. Yadra 12, 702 (2015)]

[17] E.A. Koval, O.A. Koval, Journal of Experimental and Theoretical Physics 125, 35 (2017), [Pis'ma Zh. Eksp. Teor. Fiz. 152, 45 (2017)]

[18] E.A. Koval, O.A. Koval, Physica E 93, 160 (2017)

[19] V. Melezhik, Journal of Computational Physics 92, 67 (1991) 